\title{
Optimization of pump wavelength for enhancing SC spectral broadening in silica fibers under $\mathrm{CW}$-excitation
}

\author{
L. Abrardi a , S. Martín-López ${ }^{\text {a }}$, A. Carrasco-Sanz a , F. Rodríguez-Barrios ${ }^{\text {a }}$, P. Corredera ${ }^{\text {a }}$, \\ M. L. Hernanz ${ }^{a}$ and M. González-Herráez \\ ${ }^{a}$ Instituto de Física Aplicada, CSIC, C/ Serrano 144, Madrid 28006, España; \\ ${ }^{b}$ Departamento de Electrónica, Universidad de Alcalá, 28871 Alcalá de Henares, España
}

\begin{abstract}
Supercontinuum sources generated by continuous-wave excitation are very promising for many applications as they present in general higher spectral power density then their pulsed counterparts. On the other hand, the properties of supercontinuum are very difficult to be controlled as the initial broadening is driven by modulation instability. This latter one breaks-up the $\mathrm{CW}$ radiation into a train of ultra-short pulses whose peak power, spectral length and shape strongly depend on the power, coherence and noise of the pump and on the fiber properties. In this paper, we present a preliminary work on the role of chromatic dispersion on supercontinuum spectral broadening in order to study how to optimize SC spectral width under CW regimes. By means of a home-made tunable high-power laser we induce supercontinuum generation by pumping at different dispersion values of the fiber. We show that at low injected powers the wider spectrum is obtained when pumping just above the zero-dispersion wavelength of the fiber. By contrast, for higher injected powers, wide and squared-shaped spectra can be obtained when pumping over a larger range of anomalous dispersion values. These results seem to be very promising for a number of applications requiring smooth, squared and high-power SC spectral profiles such as optical coherence tomography.
\end{abstract}

\section{INTRODUCTION}

Fiber-based supercontinuum (SC) sources find widespread applications in many different fields, and they are attracting much attention since the last few years. In particular, continuous-wave (CW) pumped SC sources seem to be of increasing interest as they present high spectral power density, short coherence lengths, and lower values of relative intensity noise (RIN) than their pulsed counterparts [1], [2]. Furthermore, CW-pumped SC generation remains object of difficult experimental investigation due to the limited availability of high-power $\mathrm{CW}$ sources. In this paper, we propose a new experimental study on the role of chromatic dispersion on SC spectral broadening in $\mathrm{CW}$-excitation regimes. This study wants to point out the relative importance of chromatic dispersion compared to the effect of intra-pulse Raman scattering on soliton formation and collisional processes. In fact, spectral broadening under $\mathrm{CW}$ excitation is initiated by modulation instability (MI) which breaks up the continuous wave into a train of soliton-like pulses when pumping in the anomalous dispersion region of the fiber. This process leads to the generation of fundamental solitons that are significantly frequency red-shifted due to intra-pulse Raman scattering. Chromatic dispersion plays a key role in the process of soliton formation because just above the zero-dispersion wavelength of the fiber $\left(\lambda_{\mathrm{ZD}}\right)$ the MI oscillations are faster and induce shorter pulse formation [1]. This leads to the generation of shorter solitons which, in their turn, undergo a larger red-shift per fiber length due to soliton self-frequency shift (SSFS) [4], [5]. Moreover, in the presence of higherorder dispersion, fundamental solitons can further enhance the spectral broadening by releasing excess energy in the form of dispersive-waves (DW) in the normal dispersion region of the fiber if their spectrum overlaps with the resonant DW frequencies [6]. For this reason, the most efficient spectral broadening was believed to occur when pumping just above the zero-dispersion wavelength of the fiber. Our results show that, in $\mathrm{CW}$ regimes, this is true only for low input powers; by contrary, when pumping beyond the Raman threshold, there is a wide range of chromatic dispersion values for which the spectral width of the supercontinuum remains almost constant. This range of values is broader as the pump power increases.

\footnotetext{
*abrardi@ifa.cetef.csic.es; phone 3491561 8806; fax 34914 117651;
} 


\section{EXPERIMENTAL SET-UP}

In order to pump the fiber at different dispersion values, we realized a home-made high-power tunable laser source operating at such a wavelength range that the nonlinear coefficient, Raman gain and losses of the fiber do not vary too much, but the dispersion does vary significantly. It is about a fiber ring laser mainly made up of two Erbium doped fiber amplifiers (EDFAs) and a tunable optical grating filter, as shown in Fig. 1. EDFA1 (EDFA-C17 provided by NetTest) has gain in the wavelength range of $1525-1570 \mathrm{~nm}$. After EDFA1, the output frequency is selected by inserting an optical grating filter (TB9 provided by JDS Uniphase). The filter provides a tuning range of $1525-1625 \mathrm{~nm}$ and a spectral width of $0.5 \mathrm{~nm}$ full width at half a maximum (FWHM). The grating filter is followed by a variable attenuator (provided by Accelink) which is used to control the power inserted into EDFA2. EDFA2 is a high-power fiber amplifier (Keopsys) working in the spectral range $1545-1570 \mathrm{~nm}$, whose output power can take values from $200 \mathrm{~mW}$ to $10 \mathrm{~W}$. A high-power isolator (OPNETI) is inserted at the output of EDFA2 whose losses are approximately $0.5 \mathrm{~dB}$. The $0.01 \%$ of the power delivered by EDFA2 is re-circulated in the cavity by means of a set of two calibrated $1 / 99$ couplers. We are able this way to generate a tunable source whose output power takes the same value for each wavelength during our measurements. Our fiber ring laser is single-mode, it presents a $0.08 \mathrm{~nm}$ FWHM line width at every wavelength in the available tuning range (i.e. from $1545 \mathrm{~nm}$ to $1570 \mathrm{~nm}$ ) and its power can take values from $500 \mathrm{~mW}$ to $7 \mathrm{~W}$. In Fig. 2 we show the laser output at different wavelengths for a total output power of $2 \mathrm{~W}$. The nonlinear medium for SC generation an $11 \mathrm{~km}$ dispersion-shifted fiber (DSF) provided by Corning. The dispersion curve of the fiber has been measured with a resolution of $2 \mathrm{~nm}$ using the phase-shift method described in [7].

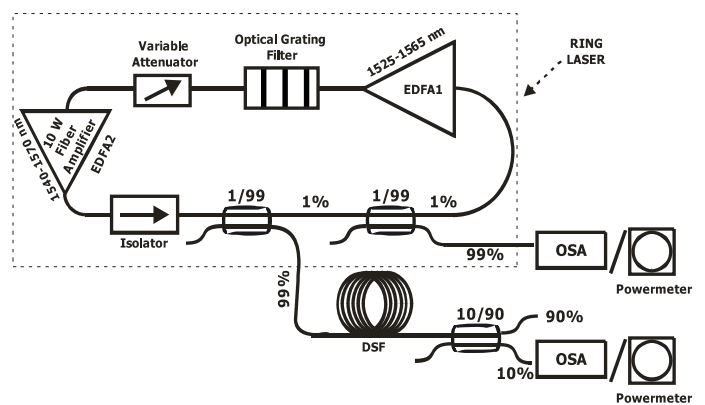

Fig.1. Experimental set-up. EDFA: Erbium-Doped Fiber Amplifier; DSF: Dispersion Shifted Fiber; OSA: Optical Spectrum Analyzer

The zero-dispersion wavelength $\lambda_{\mathrm{ZD}}$ and the nonlinear parameter $\gamma$ have been measured using a method based on modulation instability [8] and found to take values $\lambda_{\mathrm{ZD}}=1553.5 \pm 0.5 \mathrm{~nm}$, and $\gamma=1.7 \mathrm{~W}^{-1} \mathrm{~km}^{-1}$. The group-velocity dispersion (GVD) parameter $\beta_{2}$ is approximately linearly decreasing with a slope of $-0.1044 \mathrm{ps}^{2} / \mathrm{km} / \mathrm{nm}$ and it takes value $0.7828 \mathrm{ps}^{2} / \mathrm{km}$ at $\lambda=1545 \mathrm{~nm}$ and $-1.8266 \mathrm{ps}^{2} / \mathrm{km}$ at $\lambda=1570 \mathrm{~nm}$. The third and fourth-order-dispersion parameters (TOD and FOD respectively) can be considered almost constant over the whole tuning range, and they take values $\beta_{3}=0.1358 \pm 0.00377 \mathrm{ps}^{3} / \mathrm{km}$ and $\beta_{4}=(-4.8739 \pm 0.0560) \times 10^{-4} \mathrm{ps}^{4} / \mathrm{km}$ respectively. The Raman threshold is estimated to be $\mathrm{P}_{\mathrm{Cr}} \sim 1 \mathrm{~W}$ by using the Raman gain coefficient $\mathrm{g}_{\mathrm{R}}=1.5 \times 10^{-11} \mathrm{~cm} / \mathrm{W}$. The output spectrum is finally analyzed by means of an optical spectrum analyzer (OSA) with a spectral resolution of $1 \mathrm{~nm}$. The input and output power are measured by means of an integrating sphere radiometer whose responsivity is known in the whole tuning range with $1 \%$ uncertainty [9].

\section{DISPERSIVE WAVES}

In this section we want to give a deeper insight into the dynamics of soliton formation by means of a careful study of dispersive wave generation. As we mentioned, $\mathrm{CW}$-induced spectral broadening is initiated by MI which breaks-up the $\mathrm{CW}$ radiation into a train of ultra-short pulses when propagating in the region of anomalous dispersion of the fiber. As the pulses propagate, they are split into fundamental solitons [10], [11], [12] which are frequency red-shifted due to SSFS. Additionally, each fundamental soliton can, in the presence of higher-order dispersion, release excess of energy in the form of dispersive waves, enhancing the spectral broadening in the normal dispersion region of the fiber [6]. Dispersive waves are amplified only if the soliton spectrum overlaps with the resonant wave frequencies. The intensity of these waves is proportional to the intensity of the solitons at the same frequencies [6]. For this reason, more intense 
radiation is released by solitons that are closer to $\lambda_{\mathrm{ZD}}$. For this reason, the most obvious impact of chromatic dispersion on DW generation is that intense radiation is expected when pumping just above $\lambda_{\mathrm{ZD}}$. Besides, when higher pump powers are injected into the fiber, more intense DWs should in principle be generated, because higher-intensity solitons are formed. Thus, it is clear that under no other effects, the most efficient nonlinear broadening in the spectral region of normal dispersion is believed to occur at GVD parameters as close to zero as possible and for increasing injected powers. In highly non-linear fibers, under femto-second pulsed regimes, DWs arise from higher-order soliton fission. In this case, higher-order solitons are formed of soliton number $\mathrm{N}$, but they are unstable under higher-order dispersion perturbations and decay into fundamental solitons. These latter ones release excess of energy as dispersive radiation in the normal dispersion region of the fiber [13], [14], [15], [16]. Each soliton emits radiation at a frequency that satisfies the phasematching condition [6], [14], [17]:

$$
\beta\left(\omega_{D W}\right)-\beta\left(\omega_{S}\right)=\sum_{n \geq 2}^{M} \frac{\beta_{n}}{n !} \delta \omega^{n}-\gamma \frac{P_{S}}{2}=0
$$

where $\beta\left(\omega_{\mathrm{DW}}\right)$ is the wave number of the dispersive radiation and $\beta\left(\omega_{\mathrm{s}}\right)$ is the wave number of the soliton. $\delta \omega=\left(\omega_{\mathrm{DW}}\right.$ $\omega_{\mathrm{s}}$ ) is the detuning between the radiation frequency $\omega_{\mathrm{DW}}$ and the central soliton frequency $\omega_{\mathrm{s}}$. $\beta_{\mathrm{n}}$ is the $\mathrm{n}$-th dispersion coefficient calculated at $\omega_{\mathrm{s}} ; \gamma \mathrm{P}_{\mathrm{s}} / 2$ is the soliton nonlinear phase shift where $\gamma$ is the nonlinear parameter of the fiber and $\mathrm{P}_{\mathrm{s}}$ is the soliton peak power. In highly non-linear fibers under femtosecond pulse excitation, DWs significantly contribute to the total spectral broadening because new components at different frequencies are radiated by each of the $\mathrm{N}$ fundamental solitons. In The CW-regimes the same phase-matching condition has to be satisfied, but the dynamics are quite different and not completely understood.

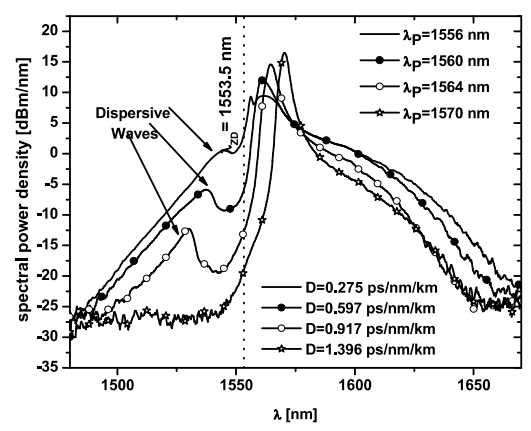

(a)

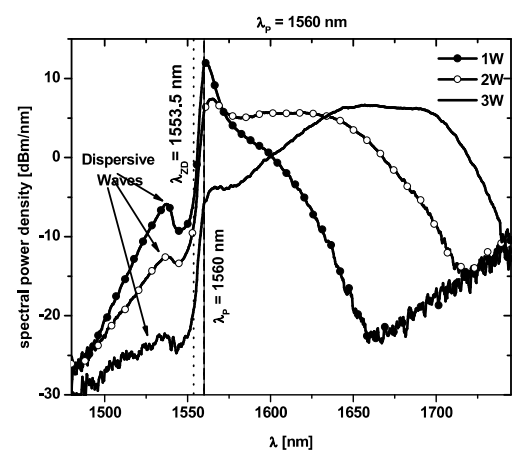

(b)

Fig.2. (a) Output spectra obtained for an input power of $1 \mathrm{~W}$. We can observe dispersive wave emission in the normal dispersion region of the fiber and decreasing spectral width when pumping further away from $\lambda_{\mathrm{ZD}}$. (b) Output spectra obtained for three different input powers at pump wavelength $\lambda_{\mathrm{P}}=1560 \mathrm{~nm}$. DWs undergo stronger attenuation with increasing input power

In fact, fundamental solitons of slightly different amplitude and time duration originate from phase noise of the pump due to MI-induced noise amplification [10], [11], [12]. In our experiment we observed DW generation, whose spectrum shows a clear local maximum in intensity, as it can be seen in Fig.2 (a). For a given input wavelength, this peak always falls at the same frequency for different input powers, as it can be seen in Fig.2 (b). For this reason, we made the hypothesis that the radiation frequency weakly depends on the soliton peak power, and we neglected the non-linear phase shift:

$$
\sum_{n \geq 2}^{M} \frac{\beta_{n}}{n !} \delta \omega^{n-2}=0
$$

This approximation is in agreement with other previous theoretical predictions [6], [18]. As we can not measure the central frequency of the generated solitons, we made the second hypothesis that most of the DWs are radiated at beginning of soliton formation, when solitons are expected to have central wavelength just above the pump wavelength. 
According to this hypothesis, we calculated the DW frequency that would be radiated by a soliton at the pump frequency by using Eq. (2) up to the fourth-order $(M=4)$ and setting $\omega_{\mathrm{s}}=\omega_{\mathrm{P}}$. We found that the frequency of the peaks of the blueshifted radiation were in good agreement with this assumption. Measurements were performed at different input powers and different pump wavelengths and are shown in Fig.3 together with the theoretical curve. Our experiment seems to confirm that most solitons are generated just above the pump wavelength, around the same central frequency. Moreover, most dispersive radiation is emitted at the first stage of soliton formation, before solitons undergo a significant SSFS.

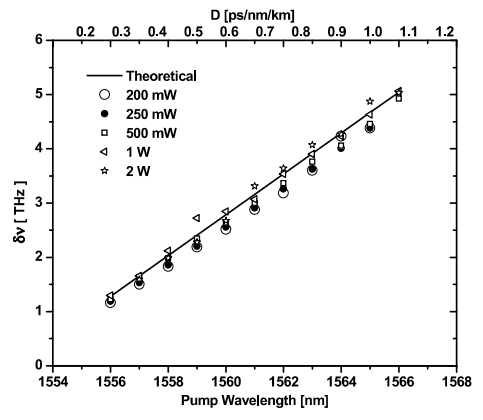

Fig.3. Frequency shift between the DW-peak frequency and the pump frequency, as function of pump wavelength.

These dynamics are not very sensitive to the average injected power until a threshold $(\mathrm{P}=3 \mathrm{~W}$ in our case) is reached for which some blue-shifted radiation is still present in the spectra, but no peaks can be seen anymore (see Fig.2 (b)). An interesting result of this study is that the presence of a well defined peak in the blue part of the spectrum seems to indicate that higher-order solitons are not generated or, at most, only solitons of low order $(\mathrm{N}<10)$ are formed. In fact, if the dispersive radiation were the result of higher-order soliton fission, a much broader and flatter blue-shifted spectrum would be generated due to the contribution of the $\mathrm{N}$-soliton decay into $\mathrm{N}$ fundamental solitons of different central frequency [13], [14]. Besides, the maximum intensity value would appear at the blue edge of the spectrum [14], [15], which is not our case. These results confirm that solitons of different amplitude and temporal width originate from phase noise of the pump due to MI, as predicted by the mentioned works [10], [11], [12]. On the other side, this model can not explain the broad spectra that are obtained. In fact, previous theoretical studies proved that MI-induced solitons have too long time duration to undergo such a significant red-shift due to SSFS [19]. For this reason, a coherent description of our results can only be given by taking into account some other effects. For this reason, we believe that our experimental results prove the latest theoretical predictions for which in $\mathrm{CW}$ and quasi-CW regimes soliton collisions take an important role in inducing shorter soliton formation [12], [20], hence a broader spectrum. We also note that at low input powers the influence of chromatic dispersion gives rise to SC spectra having a completely different shape for different pump wavelengths and powers. In particular, for an input power of $1 \mathrm{~W}$, i.e. around the Raman threshold, the spectrum is flatter and broader when the pump wavelength is just above $\lambda_{\mathrm{ZD}}$ and stronger pump depletion takes place, as it can be seen in Fig.2 (a). For input powers higher than the Raman threshold, dispersive waves start to undergo attenuation with increasing input power, as it can be seen in Fig.2 (b). In this case, however, flatter supercontinua are obtained also at longer pump wavelengths (i.e. larger values of dispersion), but contribution of DWs radiation to the total spectral broadening is decreasing with increasing input power.

\section{INFLUENCE OF CHROMATIC DISPERSION ON SC SPECTRAL BROADENING}

In this section we analyze experimentally how chromatic dispersion affects SC spectral width in high-power regimes when propagating a CW radiation into a km-long conventional DSF. In this experiment, we obtained SC spectra with very different shapes when pumping at different wavelengths (see Fig.2 (a)) and different powers (see Fig.2 (b)). In order to compare the spectra among each other, we decided to introduce a definition of SC spectral width which takes into account their different shape and their different spectral power density distribution. As in ultra-short pulse measurements a definition of Equivalent Pulse Width (EPW) is used when the pulse shape is quite complicated (see [21]), we adopted the definition of Equivalent Spectral Width (ESW) to assign a value of spectral width to each of our spectra according to the definition: 


$$
w_{e}=\frac{2}{I_{\max }} \int_{-\infty}^{+\infty} I(\lambda) d \lambda
$$

where $\mathrm{w}_{\mathrm{e}}$ is the ESW, $\mathrm{I}(\lambda)$ is the spectral power density in $\mathrm{mW} / \mathrm{nm}$, and $\mathrm{I}_{\max }$ is the maximum value of the spectrum in $\mathrm{mW} / \mathrm{nm}$. An illustration of the definition of ESW can be seen in Fig.4 (a). It is worth noticing that this definition uses the total power under the spectral curve, so that all the generated spectral components are taken into account. Moreover, a higher value of ESW is assigned to flatter spectra because they usually present a lower maximum value. This definition is very useful when spectral widths of different supercontinua have to be compared. By using the definition of ESW we study the influence of chromatic dispersion on SC spectral broadening. With the help of our tunable source we can pump in the spectral range from $1549 \mathrm{~nm}$ to $1570 \mathrm{~nm}$, where the chromatic dispersion of the fiber takes values from -0.291 $\mathrm{ps} / \mathrm{nm} / \mathrm{km}$ to $+1.396 \mathrm{ps} / \mathrm{nm} / \mathrm{km}$. We measured the ESW for different pump wavelengths and we show it in Fig.4 (b) for different input powers. As it can be seen, for input powers up around the Raman threshold (P 1 W), there is an optimum pump wavelength for which the ESW is maximum. At input powers of $0.5 \mathrm{~W}$ and $1 \mathrm{~W}$, this maximum is obtained for a pump wavelength of $1554 \mathrm{~nm}$, which is just above the $\lambda_{\mathrm{ZD}}$ of the fiber. The transition from normal to anomalous dispersion is evident by the presence of a steep increase in the spectral broadening around the $\lambda_{\mathrm{ZD}}$ of the fiber. For an input power of $2 \mathrm{~W}$ there is an optimal pump wavelength located at $1557 \mathrm{~nm}$ where the ESW is maximum, but a quite wide spectral broadening can be obtained also at other pump wavelengths around this value. Besides, the maximum ESW at this input power is higher than for $2.5 \mathrm{~W}$ and $3 \mathrm{~W}$. This is due to the fact that at this power, output spectra show a quite uniform and squared shape, and a relatively small value of the maximum intensity. This gives rise to a higher ESW in a consistent way with the definition of ESW which favors smoother and flatter profiles.

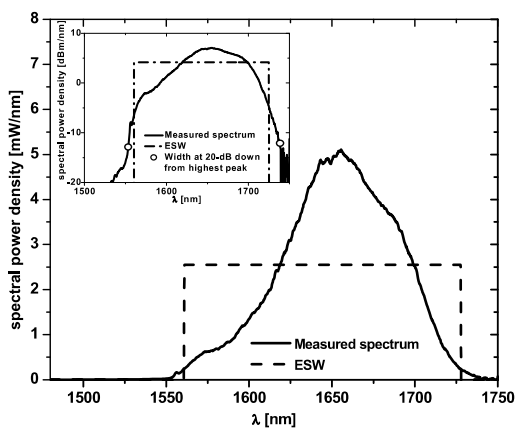

(a)

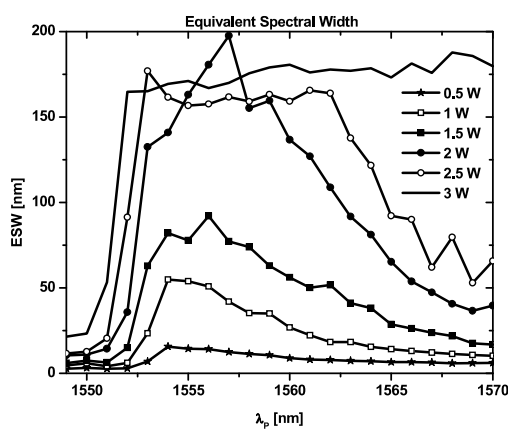

(b)

Fig.4. (a) Definition of the Equivalent Spectral Width (ESW). The calculated ESW is compared with the standard measurement 20$\mathrm{dB}$ down from the highest peak in logarithmic scale. (b) SC spectral width as function of pump wavelength for different input powers.

When powers higher than $2 \mathrm{~W}$ are injected into the fiber the behavior changes dramatically. First of all, the same value of ESW can be obtained when pumping over a larger spectral region. Second, pump depletion occurs also at longer pump wavelengths, so that flatter and square-shaped spectral profiles can be obtained for a wider range of pump wavelengths. This latter behavior, which can be clearly seen in Fig.2 (b), is probably due to the increasing influence of Raman scattering.

\section{CONCLUSIONS}

We have described an experimental study on the influence of chromatic dispersion on SC generation in km-long standard fibers under $\mathrm{CW}$-excitation regimes. This study has been done by pumping a dispersion shifted fiber with a high-power tunable $\mathrm{CW}$ laser source. By sweeping the wavelength of the laser over a small range, we can propagate the pump under very different values of chromatic dispersion. Our results show that, for input powers up to the Raman threshold, chromatic dispersion plays a fundamental role on nonlinear spectral broadening, giving rise to a broader spectrum when pumping just above the zero-dispersion wavelength of the fiber. At higher input powers, however, as soliton collisions 
and intra-pulse Raman scattering take a dominant role, the spectral width is more independent of the value of chromatic dispersion. We also show that, in this case, there is a large range of optimum pumping wavelengths for which the SC spectral width is almost constant. This range of values is increasing with increasing input power.

\section{REFERENCES}

[1] C. J. S. De Matos, S. V. Popov and J. R. Taylor, "Temporal and noise characteristics of continuous-wave-pumped continuum generation in holey fibers around 1300 nm”, Appl. Phys. Lett., vol. 85, no. 14, pp. 2706-2708, 2004.

[2] S.Martín-López,M. González-Herráez, A. Carrasco-Sanz, F. Vanholsbeeck, S. Coen, H. Fernandez, J. Solis, P. Corredera, and M. L. Hernanz, "Broad-band spectrally flat and high power density light source for fiber sensing pourposes," Meas. Sci. Technol., vol. 17, no. 5, pp. 1014-1019, 2006.

[3] A. Hasegawa, "Generation of a train of soliton pulses by induced modulation instability in optical fibers," Opt. Lett., vol. 9, no. 7, pp. 288-290, 1984.

[4] F. M. Mitschke and L. F. Mollenauer, "Discovery of the soliton self-frequency shift," Opt. Lett., vol. 11, no. 10, pp. 659-661, 1986.

[5] J. P. Gordon, “Theory of soliton self-frequency shift,” Opt. Lett., vol. 11, no. 10, pp. 662-664, 1986.

[6] N. Akhmediev and M. Karlsson, "Cherenkov radiation emitted by solitons in optical fibers," Phys. Rev. A, vol. 51, no. 3, pp. 2602-2607, 1995.

[7] B. Costa, D. Mazzoni, M. Puleo, and E. Vezzoni, "Phase-shift technique for measurement of chromatic dispersion in optical fibers using leds,” IEEE J. Quantum Elec., vol. 18, no. 10, pp. 1509-1515, 1982.

[8] C. Mazzali, D. F. Grosz, and H. L. Fragnito, "Simple method for measuring dispersion and nonlinear coefficient near the zero-dispersion wavelength of optical fibers," IEEE Photonic. Tech. L., vol. 11, no. 2, pp. 251-253, 1999.

[9] A. Carrasco-Sanz, F. Rodriguez-Barrios, P. Corredera, S. Martin-Lopez, M. Gonzalez-Herraez, and M. L. Hernanz, "An integrating sphere radiometer as a solution for high power calibrations in fiber optics," Metrologia, vol. 43, no. 2, pp. 145-150, 2006.

[10] A. Mussot, E. Lantz, H. Maillotte, T. Sylvestre, C. Finot, and S. Pitois, "Spectral broadening of a partially coherent cw laser beam in single-mode optical fibers," Opt. Express, vol. 12, no. 13, pp. 2838-2843, 2004.

[11] F. Vanholsbeeck, S. Martin-Lopez, M. Gonzalez-Herraez, and S. Coen, "The role of pump incoherence in continuous-wave supercontinuum generation," Opt. Express, vol. 13, no. 17, pp. 6615-6625, 2005.

[12] M. H. Frosz, O. Bang, and A. Bjarklev, "Soliton collision and Raman gain regimes in continuous-wave pumped supercontinuum generation,” Opt. Express, vol. 14, no. 20, pp. 9391-9407, 2006.

[13] Y. Kodama and A. Hasegawa, "Nonlinear pulse propagation in a monomode dielectric guide," IEEE J. Quantum Elect., vol. 23, no. 5, pp. 510-524, 1987.

[14] A. V. Husakou and J. Hermann, "Supercontinuum generation, four-wave mixing, and fission of higher-order solitons in photonic crystal fibers," J. Opt. Soc. Am. B, vol. 19, no. 9, pp. 2171-2182, 2002.

[15] G. Genty, M. Lehtonen, and H. L. M. Kaivola, "Enhanced bandwidth of supercontinuum generated in microstructured fibers," Opt. Express, vol. 12, no. 15, pp. 3471-3480, 2004.

[16] M. H. Frosz, P. Falk, and O. Bang, "The role of the second zero-dispersion wavelength in generation of supercontinua and bright-bright soliton-pairs across the zero-dispersion wavelength," Opt. Express, vol. 13, no. 16, pp. 6181-6192, 2005.

[17] K. M. Hilligsøe, H. N. Paulsen, J. Thøgersen, S. R. Keiding, and J. J.

Larsen, "Initial steps of supercontinuum generation in photonic crystal fibers," J. Opt. Soc. Am. B, vol. 20, no. 9, pp. 1887-1893, 2003.

[18] I. Cristiani, R. Tediosi, L. Tartara, and V. Degiorgio, "Dispersive waves generation by solitons in microstructured optical fibers," Opt. Express, vol. 12, no. 1, pp. 124-135, 2004.

${ }^{[19]}$ E. A. Golovchenko, P. V. Mamyshev, A. N. Pilipetskii, and E. M. Dianov, "Numerical analysis of the Raman spectrum evolution and soliton pulse generation in single-mode fibers,", J. Opt. Soc. Am. B, vol.8, no. 8, pp. 16261632, 1991

[20] N. Korneev, E. A. Kuzin, B. Ibarra-Escamilla, M. Bello-Jiménez, and A. Flores-Rosas, "Initial development of supercontinuum in fibers with anomalous dispersion pumped by nanosecond-long pulses," Opt. Express, vol. 16, no. 4, pp. 2636-2645, 2008.

[21] R. Trebino, Frequency-resolved optical gating: the measurement of ultrashort laser pulses. Kluwer Academic Publisher, 2002. 